\title{
Transição do modelo de ambiência em hospitais que realizam partos na Rede Cegonha
}

\author{
Transition of the environment model in hospitals that deliver \\ in Rede Cegonha
}

Dário Frederico Pasche (https://orcid.org/0000-0001-7161-8607) ${ }^{1}$

Mirela Pilon Pessatti (https://orcid.org/0000-0003-3753-4033) ${ }^{2}$

Luiza Beatriz Ribeiro Acioli de Araújo Silva (https://orcid.org/0000-0001-7095-0659) ${ }^{3}$

Maria Eliane Liegio Matão (https://orcid.org/0000-0003-1194-0324) ${ }^{4}$

Dianne Barbosa Soares (https://orcid.org/0000-0002-5149-1044) ${ }^{5}$

Ana Paula da Cruz Caramachi (https://orcid.org/0000-0002-7968-3427) ${ }^{5}$

${ }^{1}$ Instituto de Psicologia, Universidade Federal do Rio Grande do Sul. Av. Paulo Gama 110, Farroupilha. 90040-060 Porto Alegre RS Brasil. dario.pasche@gmail.com ${ }^{2}$ Curso de Arquitetura e Urbanismo, Universidade São Francisco. Campinas SP Brasil.

${ }^{3}$ Fiocruz. Rio de Janeiro RJ Brasil.

${ }^{4}$ Pontifícia Universidade Católica de Goiás. Goiânia GO Brasil.

${ }^{5}$ Ministério da Saúde. Brasília DF Brasil.
Abstract This article analyzes the environment of birth places, considering the presence of PCP room (Prepartum, Childbirth, and Postpartum) in 575 hospitals that deliver in Sistema Único de Saúde (Unified Health System) within the scope of Rede Cegonha. The data were extracted from a survey called Avaliação da atenção ao parto e nascimento nas maternidades da Rede Cegonha (Assessment of childbirth and birth care in the Rede Cegonha maternity units), carried out in 2017 by UFMA and ENSP, in partnership with the Ministry of Health. The PCP room model combines care for parturient women in a single space, favoring the role of women and the exercise of good practices in childbirth and birth care. The information was obtained by direct observation in the services, and assessment considered the presence and adequacy of PCP rooms and their distribution according to the pre-childbirth environment, which were compared with specific characteristics of these hospitals. Collective rooms for childbirths prevail and only $16.8 \%$ of beds are PCP rooms. This picture suggests difficulties in resource management, resistance to changes and insufficiencies in institutional support, which have hampered the transition from the childbirth environment model in Brazilian hospitals. The Brazilian obstetric and neonatal field has lived a fertile period, but it is necessary to build and sustain political-institutional disposition to advance the changes.

Key words "Rede Cegonha", Childbirth rooms, Childbirth, Humanization, Delivery assistance
Resumo $O$ artigo analisa a ambiência dos locais de parto, considerando a presença de quartos PPP (Pré-parto, Parto e Pós-Parto) em 575 hospitais que realizam partos no Sistema Único de Saúde no âmbito da Rede Cegonha. Os dados foram extraídos da pesquisa "Avaliação da atenção ao parto e nascimento nas maternidades da Rede Cegonha", realizada em 2017 pela UFMA e ENSP, em parceria com o Ministério da Saúde. O modelo de quarto PPP reúne o cuidado às parturientes em um só espaço, favorecendo o protagonismo das mulheres e o exercício de boas práticas de atenção ao parto e nascimento. As informações foram obtidas por observação direta nos serviços e a avaliação considerou a presença e a adequação dos quartos PPP e sua distribuição segundo ambiente de pré-parto, que foram cotejadas com características especificas desses hospitais. Prevalecem salas coletivas para os partos e apenas 16,8\% dos leitos são quartos PPP. Esse quadro sugere dificuldades de gestão dos recursos, resistências para a realização de mudanças e insuficiências no apoio institucional, que têm dificultado a transição do modelo da ambiência do parto nos hospitais brasileiros. O campo obstetriconeonatal brasileiro tem vivido um periodo fértil, mas é necessário construir e sustentar disposição político-institucional para avançar nas mudanças.

Palavras-chave Rede Cegonha, Salas de parto, Parto; Humanização, Assistência ao parto 


\section{Introdução}

O modelo brasileiro de atenção ao parto e nascimento caracteriza-se pelo uso excessivo de intervenções obstétricas e neonatais que quando empregadas sem base de evidência científica, podem estar relacionadas a desfechos maternos e perinatais desfavoráveis ${ }^{1}$. Entre as várias iniciativas para modificar este modelo, nos anos 2000, o Ministério da Saúde (MS) criou o Programa de Humanização no Pré-natal e Nascimento (PHPN). A humanização da assistência ao parto engloba várias dimensões, entre elas a ambiência, estabelecida como uma das diretrizes da Política Nacional de Humanização ${ }^{2}$ (PNH). No campo da atenção ao parto e ao nascimento, a ambiência compreende a transformação do espaço hospitalar em um ambiente acolhedor e favorável à implementação de boas práticas de atenção obstétrica e com a participação ativa das usuárias. O conceito valoriza o ambiente como contribuinte de mudanças nos processos e nas relações de trabalho pautadas na construção coletiva e participativa.

A partir desta concepção novos padrões de funcionamento dos Serviços de Atenção Obstétrica e Neonatal foram instituídos na Resolução no 36 da ANVISA, de 03/06/2008 ${ }^{3}$ (RDC 36/2008), e posteriormente incorporados a estratégia da Rede Cegonha (RC) ${ }^{4}$, lançada em 2011.

Dentre as novas estruturas introduzidas foi estabelecida a transformação dos ambientes de parto, tradicionalmente compostos por salas compartilhadas por várias mulheres, em quarto PPP (Pré-parto, Parto e Pós-Parto), espaço privativo para o trabalho de parto, parto e puerpério imediato, com banheiro anexo, dimensões suficientes para a movimentação da parturiente e uso de métodos não farmacológicos de alívio da dor e para a presença de acompanhante. Este ambiente além de estimular a superação do modelo tradicional, que artificialmente segmenta o parto em estágios de pré-parto, parto e pós-parto, propicia uma atenção diferenciada com o propósito de garantir à mulher condições de escolha de diversas posições no trabalho de parto, protagonismo e autonomia ${ }^{5}$.

Com o intuito de transformar a realidade brasileira de instalações deficientes nos serviços de saúde que realizam partos e, por muitas vezes, inadequadas para prestar atendimento de qualidade $^{6}$, inúmeras iniciativas do Ministério da Saúde foram desenvolvidas no processo de implementação da RC. Além de disponibilizar recursos financeiros para a adequação da ambiência foram adotadas medidas para facilitar a gestão dos recursos, a exemplo da transferência fundo a fundo de recursos de investimento ${ }^{7}$; a adoção do Regime Diferenciado de Contratações Públicas para obras e serviços de engenharia no âmbito do SUS'; e a criação do SISMOB - Sistema de Monitoramento de Obras. Ao mesmo tempo, o MS disponibilizou projetos arquitetônicos que poderiam ser adotados pelos gestores e realizou processos de formação de arquitetos e engenheiros das secretarias de saúde, ampliando a difusão do conceito de ambiência?.

Pesquisas sobre a ambiência dos locais de parto são exíguas e não existem estudos que tratem especificamente do conceito de ambiência em obstetrícia $^{10}$ É nesse contexto e cenário que este artigo se insere, buscando contribuir para o debate sobre a qualificação da atenção obstétrica a partir da disponibilização e da adequação de quartos PPP no Brasil e nas grandes regiões, em hospitais que realizam partos no SUS no âmbito da RC. Os achados da pesquisa foram analisados à luz de reflexões que têm sido feitas no Campo da Saúde Coletiva, na perspectiva de melhor compreender o quadro atual da ambiência dos locais de parto.

\section{Métodos}

É uma pesquisa de avaliação normativa com delineamento qualitativo e quantitativo e emprego da técnica de Estimativa Rápida Participativa. Foram elegíveis todos os hospitais públicos e privados que, em 2015, localizavam-se em região de saúde com Plano de Ação da RC, totalizando 606 estabelecimentos distribuídos em todos os estados do Brasil. A coleta de dados foi realizada em 2016/2017.

Foram utilizados três métodos de coleta de dados: 1 - entrevista pessoal com informanteschave: gestores; profissionais de saúde e puérperas para verificar a percepção quanto ao modelo de gestão e à atenção ao parto e nascimento; 2 análise documental verificou a disponibilidade de normas, protocolos e de indicadores de processo e resultados da assistência ao parto e nascimento. Dados sobre o cuidado hospitalar foram extraídos do prontuário da mulher e do RN; e 3 - observação in loco coletou dados sobre os processos de cuidado, as condições de infraestrutura, a planta física, os materiais, os equipamentos e por meio de questionário específico contaram os leitos obstétricos e neonatais disponíveis. $\mathrm{O}$ instrumento desenvolvido se baseou nas RDC36 $6^{3}$ RDC50 ${ }^{11} \mathrm{e}$ Portaria 930/2012 $2^{12}$. A avaliadora, acompanhada de um funcionário designado pela direção do 
hospital e na maioria das vezes com a presença de um representante da Secretaria Estadual de Saúde e/ou da Secretaria Municipal de Saúde, percorreu todos os ambientes da maternidade, desde a porta de entrada e recepção até o alojamento conjunto, incluindo a unidade neonatal. Ao final da observação in loco, o questionário de contagem de leitos obstétricos e neonatais era assinado pela avaliadora, pelo funcionário do hospital e pelo representante da secretaria de saúde. Para a presente análise, são utilizadas somente as informações coletadas na observação in loco.

A equipe de pesquisa incluía a coordenação nacional composta por pesquisadores da Escola Nacional de Saúde da Fundação Oswaldo Cruz, Universidade Federal do Maranhão e técnicos do MS da área técnica da Saúde da Mulher. Cada estado brasileiro teve um coordenador que participou da organização do trabalho de campo e da seleção da equipe de avaliadores da pesquisa do estado. Ao todo foram 107 avaliadores distribuídos pelo país. Todos eram profissionais de saúde com experiência de trabalho em maternidades. A capacitação das 27 equipes estaduais de pesquisa foi realizada localmente, de forma padronizada, durante cinco dias consecutivos, incluindo leitura do instrumento do questionário, aplicação prática do questionário em hospitais e envio dos dados coletados ao REDCap. Mais informações em Vilela at al. ${ }^{13}$.

Os hospitais foram classificados segundo as grandes regiões do país, localização (capital, fora da capital), natureza jurídica do estabelecimento (público e privado) e tipo de gestão (municipal, estadual e dupla). Como estabelecimentos privados foram classificados aqueles que constavam no Cadastro Nacional de Estabelecimentos de Saúde nas seguintes categorias: entidade empresarial; sem fins lucrativos; pessoa física. Os hospitais foram classificados: segundo o volume de partos registrados no Sistema de Informação sobre Nascidos Vivos (Sinasc), categorizado em baixo (até 999 partos por ano), médio (de 1.000 a 2.999 por ano) e alto (de 3.000 e mais partos por ano); a existência de UTI de neonatal; e de ser de referência para parto de alto risco ${ }^{14}$.

Dentre os requisitos de estrutura exigidos pela legislação brasileira foi verificado o tipo de ambientes de pré-parto ofertado (sala coletiva sem separação entre os leitos; sala coletiva com separação por cortinas; quarto PPP e/ou os dois tipos de ambientes). Também foi calculado o percentual de oferta de quarto PPP entre os leitos de trabalho de parto. Ademais, foi examinada a existência de banheiro exclusivo com chuveiro e água quente diretamente ligado ao quarto PPP e de pelo menos um equipamento (bola bobath, cavalinho, escada de ling, etc.) para alívio não farmacológico da dor.

A análise compreendeu a distribuição de frequência relativa das variáveis estudadas segundo grandes regiões. Por último, os dados sobre as características dos hospitais foram observados segundo a presença de pelo menos um quarto PPP no hospital avaliado.

A pesquisa atende a Resolução no. 196/96 do Conselho Nacional de Saúde e foi aprovada pelo Comitê de Ética em Pesquisa com Seres Humanos da Universidade Federal do Maranhão e da Escola Nacional de Saúde Pública Sérgio Arouca, em 14 de dezembro de 2016. Todos os cuidados foram adotados visando a garantir o sigilo e confidencialidade das informações.

\section{Resultados}

A presente análise compreende 575 hospitais (95,0\% do total de avaliados). Na Tabela 1 observa-se que do total de estabelecimentos de saúde investigados, 37,4\% estão localizados na região Sudeste, seguida da Nordeste com $28,9 \%$, Norte e Sul com $13,0 \%$ e o Centro-Oeste (6,6\%). Quanto à natureza jurídica, $57,7 \%$ eram públicos, e os demais privados $(42,3 \%)$. Ao analisar por grandes regiões observa-se que no Norte quase dois terços do total dos hospitais eram públicos, valor que se reduz para em torno de $60 \%$ nas regiões Nordeste, Sudeste e Centro-oeste e compreende um pouco mais de um terço na região Sul. Notase que para as regiões Norte, Nordeste e Sudeste em torno de $30 \%$ dos estabelecimentos estão localizados na capital, entretanto o padrão se altera, para as regiões Centro-oeste e Sul, sendo que na primeira a maior parte dos hospitais está localizada nas capitais $(63,2 \%)$, já no Sul engloba 16,5\%. Em relação ao tipo de gestão, é marcante a predominância de estabelecimentos municipais $(65,7 \%)$, sendo que na região Sul também se destaca a gestão dupla em 26,6\% dos hospitais. Para o país predominam os estabelecimentos com volume de parto médio (entre 1.000 e 2.999 partos ao ano), com pouca variação entre as grandes regiões. Observa-se, ainda na Tabela 1 , que hospitais com disponibilidade de leitos de UTI Neonatal variaram de $28,6 \%$ na região Nordeste até $69,3 \%$ no Sudeste. No Brasil, em torno de um quinto dos hospitais é referência para o alto risco, sendo o menor percentual encontrado na Região Norte $(7,8 \%)$ e o maior na Região Sudeste $(28 \%)$. 
Na Tabela 2 observa-se que em mais de dois terços dos hospitais do país o trabalho de parto é realizado em sala coletiva, valor que se elevou para as regiões Norte $(80,6 \%)$, Nordeste $(77,7 \%)$ e Sul (76,0\%). A sala coletiva sem separação entre os leitos prevalece no Norte $(49,4 \%)$ e no Nordeste $(45,8 \%)$. A disponibilidade exclusiva de quarto PPP não atinge $12 \%$ dos hospitais avaliados, é praticamente inexistente na Região Sul (2,5\%), sendo o melhor cenário encontrado na Região Centro-Oeste, com 26,3\%. Em 17,2\% dos hospitais são adotados os dois tipos de ambientes, a maior presença é verificada na Região Sudeste $(27,4 \%)$, e as menores no Norte $(3,9 \%)$ e Nordeste $(9,0 \%)$.

Na Tabela 3 observa-se que 3.358 leitos são destinados para o trabalho de parto. Em média cada maternidade tem 5,8 leitos para o trabalho de parto, variando de 4,7 leitos na Região Norte até 6,7 na Região Nordeste. A proporção de quartos PPP foi de $16,8 \%$ do total de leitos para o trabalho de parto, sendo a menor frequência

Tabela 1. Número de maternidades visitadas, frequência relativa dos estabelecimentos de saúde, segundo localização na capital e características de complexidade. Brasil e grandes regiões.

\begin{tabular}{|c|c|c|c|c|c|c|}
\hline & \multicolumn{6}{|c|}{ Região } \\
\hline & Norte & Nordeste & Sudeste & Sul & $\begin{array}{l}\text { Centro- } \\
\text { Oeste }\end{array}$ & Brasil \\
\hline \multicolumn{7}{|l|}{ Hospitais } \\
\hline Número & 77 & 166 & 215 & 79 & 38 & 575 \\
\hline Porcentagem & 13,4 & 28,9 & 37,4 & 13,7 & 6,6 & 100,0 \\
\hline Localização na capital & 26,0 & 30,7 & 27,9 & 16,5 & 63,2 & 29,2 \\
\hline \multicolumn{7}{|l|}{ Natureza jurídica ${ }^{1}$} \\
\hline Público & 72,7 & 60,2 & 58,1 & 34,2 & 63,2 & 57,7 \\
\hline Privado & 27,3 & 39,8 & 41,9 & 65,8 & 36,8 & 42,3 \\
\hline \multicolumn{7}{|l|}{ Tipo de gestão $^{1}$} \\
\hline Municipal & 61,0 & 67,5 & 72,6 & 49,4 & 63,2 & 65,7 \\
\hline Estadual & 33,8 & 24,1 & 22,8 & 24,1 & 34,2 & 25,6 \\
\hline Dupla & 5,2 & 8,4 & 4,2 & 26,6 & 2,6 & 8,5 \\
\hline \multicolumn{7}{|l|}{ Volume de partos ${ }^{2}$} \\
\hline Até 999 partos & 28,6 & 19,9 & 20,1 & 24,1 & 13,2 & 21,3 \\
\hline 1000 a 2999 & 45,5 & 47,0 & 51,9 & 53,2 & 52,6 & 49,8 \\
\hline 3000 e mais & 26,0 & 33,1 & 28,0 & 22,8 & 34,2 & 28,9 \\
\hline Com Unidade de Terapia Intensiva Neonatal ${ }^{3}$ & 28,6 & 34,3 & 69,3 & 54,4 & 52,6 & 50,6 \\
\hline Referência para alto risco ${ }^{4}$ & 7,8 & 18,7 & 28,4 & 17,7 & 21,6 & 20,9 \\
\hline
\end{tabular}

Fontes: 1. Cnes, 2017; 2. Sinasc 2017; 3 Contagem de Leito; 4. Informação fornecida pelo MS.

Tabela 2. Distribuição das maternidades segundo ambiente de pré-parto. Brasil e grandes regiões

\begin{tabular}{|c|c|c|c|c|c|c|c|c|c|c|c|c|}
\hline \multirow[b]{2}{*}{ Ambientes de Pré Parto } & \multicolumn{2}{|c|}{ Norte } & \multicolumn{2}{|c|}{ Nordeste } & \multicolumn{2}{|c|}{ Sudeste } & \multicolumn{2}{|c|}{ Sul } & \multicolumn{2}{|c|}{$\begin{array}{c}\text { Centro- } \\
\text { Oeste }\end{array}$} & \multicolumn{2}{|c|}{ Brasil } \\
\hline & $\mathbf{N}$ & $\%$ & $\mathbf{N}$ & $\%$ & $\mathbf{N}$ & $\%$ & $\mathbf{N}$ & $\%$ & $\mathbf{N}$ & $\%$ & $\mathbf{N}$ & $\%$ \\
\hline $\begin{array}{l}\text { Sala coletiva sem separação } \\
\text { entre os leitos }\end{array}$ & 38 & 49,4 & 76 & 45,8 & 37 & 17,2 & 16 & 20,3 & 7 & 18,4 & 174 & 30,3 \\
\hline $\begin{array}{l}\text { Sala coletiva com separação } \\
\text { com cortinas / box }\end{array}$ & 24 & 31,2 & 53 & 31,9 & 87 & 40,5 & 44 & 55,7 & 13 & 34,2 & 221 & 38,4 \\
\hline $\begin{array}{l}\text { Quarto PPP (pré parto, } \\
\text { parto e pós parto) }\end{array}$ & 8 & 10,4 & 17 & 10,2 & 30 & 14,0 & 2 & 2,5 & 10 & 26,3 & 67 & 11,7 \\
\hline Dois tipos de ambientes & 3 & 3,9 & 15 & 9,0 & 59 & 27,4 & 16 & 20,3 & 6 & 15,8 & 99 & 17,2 \\
\hline Outros $^{*}$ & 4 & 5,2 & 5 & 3,0 & 2 & 0,9 & 1 & 1,3 & 2 & 5,3 & 14 & 2,4 \\
\hline
\end{tabular}

${ }^{*}$ Centro cirúrgico reservado para parto; quartos duplos, na própria enfermaria; sala de observação.

Fonte: Instrumento de coleta de dados: Contagem de leitos e Roteiro de Observação. 
verificada na Região Sul (8\%) e a maior na Região Centro-Oeste (31\%). As regiões Sul (14,3\%) e Norte $(22,2 \%)$ apresentaram os menores percentuais de quartos PPP que possuem banheiro exclusivo com água quente no chuveiro. A disponibilidade de equipamentos para alívio não farmacológico da dor foi encontrada em 91,9\% dos quartos PPP com pouca variação entre as regiões.
A Tabela 4 evidencia que os hospitais com pelo menos um quarto PPP apresentam maior frequência nas capitais $(37,5 \%)$, entre os hospitais com gestão estadual $(27,5 \%)$, que realizam mais de 3000 partos por ano $(38,0 \%)$, e de maior complexidade medida pela presença de UTI Neonatal $(34,0 \%)$ e ser referência para gestante de alto risco $(34,2 \%)$.

Tabela 3. Número de maternidades, leitos de trabalho de parto, quartos PPP e aspecto de estrutura do quarto PPP.

\begin{tabular}{|c|c|c|c|c|c|c|}
\hline & \multicolumn{6}{|c|}{ Região } \\
\hline & Norte & Nordeste & Sudeste & Sul & $\begin{array}{l}\text { Centro- } \\
\text { Oeste }\end{array}$ & Brasil \\
\hline Número de maternidades & 77 & 166 & 215 & 79 & 38 & 575 \\
\hline Total de leitos de trabalho de parto & 360 & 1106 & 1260 & 403 & 229 & 3358 \\
\hline Média de leitos de trabalho de parto & 4,7 & 6,7 & 5,9 & 5,1 & 6,0 & 5,8 \\
\hline Total de quartos PPP & 46 & 142 & 275 & 31 & 71 & 565 \\
\hline Número de quartos PPP avaliados & 45 & 100 & 206 & 28 & 55 & 434 \\
\hline Percentual & 97,8 & 70,4 & 74,9 & 90,3 & 77,5 & 76,8 \\
\hline $\begin{array}{l}\text { Possui banheiro exclusivo diretamente } \\
\text { ligado ao quarto com chuveiro com }\end{array}$ & 10 & 71 & 196 & 4 & 30 & 311 \\
\hline Percentual & 77,8 & 70,3 & 95,1 & 85,7 & 56,6 & 73,5 \\
\hline $\begin{array}{l}\text { Equipamentos para alívio não } \\
\text { farmacológico da dor }\end{array}$ & 43 & 96 & 184 & 26 & 50 & 399 \\
\hline Percentual & 95,6 & 96,0 & 89,3 & 92,9 & 90,9 & 91,9 \\
\hline
\end{tabular}

Tabela 4. Distribuição das maternidades com quartos PPP por localização, natureza jurídica, tipo de gestão, volume de partos e nível de complexidade. Brasil e grandes regiões.

\begin{tabular}{lcccc}
\hline & \multicolumn{3}{c}{ Maternidades com Quartos PPP } \\
\cline { 2 - 6 } & \multicolumn{3}{c}{ Não } & \multicolumn{3}{c}{ Sim } \\
\hline Localização da maternidade & No & $\%$ & $\mathbf{N}^{\circ}$ & $\%$ \\
$\quad$ Capital & 105 & 62,5 & 63 & 37,5 \\
$\quad$ Interior & 333 & 81,8 & 74 & 18,2 \\
Natureza jurídica & & & & \\
$\quad$ Administração Pública & 250 & 75,3 & 82 & 24,7 \\
$\quad$ Entidade empresarial/ Sem fins lucrativos/ Pessoa Física & 188 & 77,4 & 55 & 22,6 \\
Volume de partos & & & & \\
$\quad$ Até 999 partos & 104 & 85,2 & 18 & 14,8 \\
1000 a 2999 & 231 & 80,8 & 55 & 19,2 \\
$\quad 3000$ e mais & 103 & 62,0 & 63 & 38,0 \\
Com Unidade de Terapia Intensiva & & & & \\
$\quad$ Sim & 192 & 66,0 & 99 & 34,0 \\
$\quad$ Não & 246 & 86,6 & 38 & 13,4 \\
Referência para gestante de alto risco & & & & \\
$\quad$ Sim & 79 & 65,8 & 41 & 34,2 \\
$\quad$ Não & 359 & 79,1 & 95 & 20,9 \\
\hline
\end{tabular}




\section{Discussão}

O panorama da disponibilidade e da adequação de quarto PPP é um dos aspectos importantes na avaliação das potencialidades do sistema de saúde em responder às necessidades de atenção de mulheres e recém-nascidos durante o parto e o nascimento.

Embora os processos envolvidos na assistência ao parto e nascimento das maternidades selecionadas não tenham sido considerados neste artigo, as evidências da associação entre ambientes adequados no cuidado seguro à mulher e ao recém-nascido e a ocorrência de resultados favoráveis reafirmam a importância da avaliação da estrutura, mesmo de forma isolada ${ }^{15}$. Os achados da pesquisa, tomando o quarto PPP como modelo de atenção ao parto, mostram que a adequação da ambiência do parto se apresenta como um importante passivo para a qualificação da atenção obstétrica brasileira, sobretudo na perspectiva de ampliação da frequência de boas práticas, muitas vezes impossibilitadas de serem exercidas sem constrangimento em salas coletivas.

A transição do modelo de ambiência do parto e nascimento que segmenta e separa em ambientes específicos o pré-parto e o parto, para o modelo quarto PPP, representa ainda um enorme desafio, em que pese mais de uma década de vigência da RDC 36/2008 3 e quase uma da Rede Cegonha $^{4}$. A presença de salas coletivas é uma realidade na maioria dos hospitais avaliados e ainda é tímida a adoção exclusiva de quarto PPP. Uma parcela também pequena de hospitais adota modelos mistos, iniciando algum processo de mudança da ambiência. Os hospitais da Rede Cegonha foram responsáveis por quase metade dos partos realizados no país e $61,2 \%$ dos partos do SUS em $2017^{16}$. É provável que os hospitais públicos e/ou conveniados ao SUS não cobertos pelo Plano de Ação da RC apresentem ainda menor disponibilidade de quartos PPP.

Os resultados apontam que um número significativo dos hospitais avaliados não possui banheiro exclusivo com água quente no chuveiro, indicativo de que uma parcela importante das mulheres não teve assegurado o acesso a essa tecnologia, nem à sua privacidade, aspecto fundamental para o bom desenrolar da fisiologia feminina. A presença de equipamentos para alívio não farmacológico da dor se verifica na maioria dos hospitais, o que mostra que mesmo a ambiência do parto não sendo adequada, esforços de incorporação desses equipamentos e tecnologias de cuidado têm sido realizados ${ }^{17}$.
Os hospitais localizados em capitais, em geral com maior volume de parto, com UTI Neonatal e de referência para a gestação de alto risco apresentaram um maior número de maternidades com pelo menos um quarto PPP instalado. Observação semelhante foi apontada por Magluta et al. ${ }^{18}$ e Bittencourt et al. ${ }^{6}$ que constataram que os hospitais de maior complexidade apresentam melhores níveis de adequação nas dimensões de estrutura avaliadas. Nosso estudo tem a vantagem de que os dados utilizados se basearam na observação direta de avaliadores treinados, reduzindo assim, as incertezas quanto à confiabilidade dos dados coletados.

Embora o estudo sobre a ambiência do parto tenha se restringido à verificação da estrutura dos serviços de saúde e não tenha verificado as razões que tornam a transição da ambiência do parto praticamente estagnada no país, é importante compreender os diferentes aspectos envolvidos e, assim, apontar mesmo que de forma limitada, alternativas para modificar a permanência do modelo tradicional de atenção ao parto.

A baixa disponibilidade de quarto PPP e seus diferentes estágios de implantação segundo grandes regiões pode estar associada ao contexto de subfinanciamento estrutural do SUS e, mais recentemente, ao teto e corte de gastos públicos ${ }^{19}$, que pode ter inviabilizado a execução de reformas dos espaços físicos das maternidades, assim como também as dificuldades dos gestores na execução dos recursos financeiros liberados pelo MS para a adequação da ambiência.

Dados do Ministério da Saúde ${ }^{20}$ demonstram que os recursos liberados, sobretudo até o ano de 2015, não foram totalmente executados pelas esferas subnacionais e os recursos captados demoraram para se transformar em novos serviços e em espaços reformados. No período compreendido entre os anos 2011-2018 foram aprovadas e empenhadas pelo MS 481 propostas de obras nos componentes Centro de Parto Normal (CPN), Casa da Gestante, do Bebê e da Puérpera (CGBP), ambiência dos serviços de atenção ao parto e nascimento, construções e ampliações de novas maternidades e estruturação de unidades de atenção especializada. Destas propostas, 211 (44\%) eram referentes à ambiência, perfazendo o montante de $\mathrm{R} \$ 51.878 .795,02^{20}$. Até o final de abril de 2019, considerando as propostas vigentes (empenhadas e não canceladas pelo MS), apenas 46 delas (21\%), foram finalizadas. Entre as 124 propostas aprovadas no ano de 2013, apenas 59\% (46 obras) foram finalizadas até final de 2018, decorrido, então, um período de 5 anos. $\mathrm{O}$ 
número reduzido de projetos apresentados indica, também, que a transformação das práticas de atenção às mulheres e às crianças não tem sido tomada como uma prioridade, logo não haveria razões para se realizar investimentos no ambiente do parto $^{21}$

É reconhecida a resistência às mudanças ${ }^{22}$ por parte de dirigentes e equipes dos serviços de saúde para preservar aquilo que se institucionalizou como cultura de cuidado na organização. Em cenários mais endurecidos, onde as relações de saber-poder são mais assimétricas, às mudanças tendem a conformar forças em torno da conservação, perpetuando uma série de situações, como a continuidade de realização de intervenções desnecessárias, preteridas por aquelas sabidamente benéficas $^{23}$. No entanto, é importante destacar que embora a modificação do ambiente tenha alcançado uma cobertura restrita, foram observados avanços no modelo de atenção preconizado pela RC conforme destacaram artigos que compararam a evolução das boas práticas e intervenções desnecessárias na assistência à mulher e ao RN nas mesmas maternidades do SUS em 2011 e a atual avaliação da RC de 2016/201724,25. Tais movimentos de mudança nos modos de cuidar convivem no mesmo tempo histórico com a presença de práticas inadequadas de atenção ao parto e nascimento, colocando o Brasil diante de um quadro paradoxal, que mistura inovação e conservação conformando, muitas vezes, processos híbridos que amalgamam elementos de conservação-transformação.

Alterações nos modelos de cuidado em saúde não decorrem ato contínuo à introdução de novas regras no trabalho, nem mesmo de estratégias governamentais de natureza sistêmica, como a RC. Todavia, as chances de sustentação das inovações emergentes dessas formulações são maiores quando resultam de processos coletivos de negociação nos ambientes do trabalho ${ }^{26}$, que se traduziriam em protocolos e regulamentos técnicos, assim como em projetos de modificação da ambiência para o parto sustentados em uma nova ética do trabalho.

Foi a partir desse entendimento que o apoio institucional preconizado pela RC tomou o Método da $\mathrm{PNH}^{27}$,- inclusão dos sujeitos e dos efeitos dessa inclusão - decorrendo daí a materialização de princípios e diretrizes em dispositivos, que são tecnologias de trabalho. Tratar-se-ia da implementação de um modelo de ação institucional que permitisse a produção de consensos sobre modos de gerir e de cuidar que resultassem em novos contratos de trabalho, em novos modos de fazer, em novos espaços de trabalho. Assim, o processo de implementação da RC contou com a participação de apoiadores, grupos condutores da RC e coordenações técnicas das secretarias estaduais e municipais de saúde, que passaram a subsidiar as equipes das maternidades na introdução e na sustentação de mudanças nos processos de planejamento, avaliação e nas agendas de trabalho, conforme evidenciado no Caderno HumanizaSUS ${ }^{28}$. Além de corroborar com a formação de arquitetos e engenheiros ${ }^{9}$ comprometidos com o desenvolvimento de Projetos Cogeridos de Ambiência ${ }^{29}$, onde os processos de cuidado e as necessidades de mudanças nos ambientes de trabalho são delimitados pelo conceito de ambiência.

Esse modo intensivo ${ }^{30}$ de atuar dos apoiadores em processos formativos e na mobilização de equipes gestoras e de trabalhadores, por certo não se fez capilarizado em todo o SUS. Alcançá-lo por um modo de ação intensivo coloca a questão das estratégias e do tempo político de sua realização. Em muitos serviços de saúde os efeitos intensivos foram importantes, mas em outros é provável que a RC, como uma nova ética do cuidado, nem tenha chegado, fazendo permanecer inalteradas as ambiências e os modos de cuidar. E mesmo naqueles em que se alcançou o apoio, os relatos são de dificuldades para se implementar mudanças ${ }^{28}$.

Modificações na ambiência demandam conjugar esforços para que as mudanças 'no estado das coisas' acionem processos de alterações nos modelos de atenção em consonância com os pressupostos da humanização do parto e do nascimento. Esses processos, todavia, são de natureza distinta e a 'transformação das coisas' pari passo à 'reforma das pessoas' ${ }^{31}$ demanda tempo e condições para a emergência de novos processos de subjetivação. Assim, é necessário construir e sustentar disposição político-institucional para alterar dinâmicas de trabalho, que por sua vez convocam reposicionamentos subjetivos, dada a natureza ontogenética do trabalho humano ${ }^{32}$. É nessa medida que compreendemos, em parte, as dificuldades para se implementar mudanças no modelo de ambiência dos hospitais que realizam partos pelo SUS.

Essas questões deveriam ser compreendidas como desafios para a comunidade científica brasileira, as organizações profissionais, os gestores do SUS, do MS e do Ministério da Educação e para o conjunto da sociedade brasileira. Ultrapassar essa realidade, na qual a ambiência inadequada é analisadora, porque a explicita, pressupõe avançar culturalmente na direção da 
conformação de um novo projeto social, no qual a vida, o viver pleno sejam as referências éticas imperativas.

Todavia, mesmo diante desse cenário de uma transição lenta da ambiência do parto, o campo obstetriconeonatal brasileiro tem vivido um "período fértil e promissor no que se refere à possibilidade de revisão de conceitos, valores e (...) práticas assistenciais" ${ }^{33}$, o que já pode ser constatado por Leal et al. ${ }^{17}$, que apontam para um aumento "significativo do acesso à tecnologia apropriada ao parto e nascimento (...) com aumento da proporção de uso de práticas benéficas e redução de práticas consideradas prejudiciais". A experimentação de novos modos de gerir e cuidar no campo obstetriconeonatal é um importante espaço para se construir as bases de um projeto social que coloque a vida em seu centro. As experiências brasileiras já nos mostram isso e mostram também que políticas públicas como a Rede Cegonha são apostas e conquistas fundamentais para a melhoria da saúde de mulheres e crianças.

\section{Colaboradores}

DF Pasche participou da concepção, elaboração e coordenação do artigo. MP Pessatti trabalhou na concepção e na redação e revisão final do artigo. LBRAA Silva trabalhou concepção do artigo, coleta de dados e revisão do artigo. MEL Matão, DB Soares e APC Caramachi trabalharam na concepção do artigo, participaram coleta de dados e da revisão bibliográfica.

\section{Agradecimentos}

A Esther Vilela e Sonia Duarte de Azevedo Bittencourt pelas generosas contribuições no processo de discussão, que trouxeram elementos enriquecedores para a abordagem e análise do tema. 


\section{Referências}

1. Leal MC, Pereira APE, Domingues RMSM, Theme Filha MM, Dias MAB, Nakamura-Pereira M, Bastos MH, Gama SGN. Intervenções obstétricas durante o trabalho de parto e parto em mulheres brasileiras de risco habitual. Cad Saude Publica 2014; 30(Supl. 1):17-32.

2. Brasil. Ministério da Saúde (MS). HumanizaSUS: Documento base para gestores e trabalhadores do SUS. Brasília: Editora do MS; 2010.

3. Agência Nacional de Vigilância Sanitária (Anvisa). Resolução RDC n 36, de 3 de junho de 2008. Dispõe sobre Regulamento Técnico para Funcionamento dos Serviços de Atenção Obstétrica e Neonatal. Diário Oficial da União 2008; 4 jun.

4. Brasil. Ministério da Saúde (MS). Portaria no 1.459, de 24 de junho de 2011. Diário Oficial da União 2011; 27 jun.

5. Brasil. Ministério da Saúde (MS). Orientações para elaboração de projetos arquitetônicos Rede Cegonha: ambientes de atenção ao parto e nascimento [recurso eletrônico]. Brasília: MS; 2018.

6. Bittencourt SDA, Reis LGC, Ramos MM, Rattner D, Rodrigues PL, Neves DCO, Arantes SL, Leal MC. Estrutura das maternidades: aspectos relevantes para a qualidade da atenção ao parto e nascimento. Cad Saude Publica 2014; 30(Supl. 1):S208-S219.

7. Brasil. Lei Complementar $n^{\circ} 141$, de 13 de janeiro de 2012. Regulamenta o $\$ 3$ o do art. 198 da Constituição Federal para dispor sobre os valores mínimos a serem aplicados anualmente pela União, Estados, Distrito Federal e Municípios em ações e serviços públicos de saúde; estabelece os critérios de rateio dos recursos de transferências para a saúde e as normas de fiscalização, avaliação e controle das despesas com saúde nas 3 (três) esferas de governo; revoga dispositivos das Leis nos 8.080, de 19 de setembro de 1990, e 8.689, de 27 de julho de 1993; e dá outras providências. Diário Oficial da União 2012; 16 jan.

8. Brasil. Lei $\mathrm{n}^{\circ} 12.462$, de 4 de agosto de 2011. Institui o Regime Diferenciado de Contratações Públicas - RDC; altera a Lei $\mathrm{n}^{\circ} 10.683$, de 28 de maio de 2003, que dispõe sobre a organização da Presidência da República e dos Ministérios, a legislação da Agência Nacional de Aviação Civil (Anac) e a legislação da Empresa Brasileira de Infraestrutura Aeroportuária (Infraero); cria a Secretaria de Aviação Civil, cargos de Ministro de Estado, cargos em comissão e cargos de Controlador de Tráfego Aéreo; autoriza a contratação de controladores de tráfego aéreo temporários; altera as Leis $\mathrm{n}^{\circ} \mathrm{S} 11.182$, de 27 de setembro de $2005,5.862$, de 12 de dezembro de 1972, 8.399, de 7 de janeiro de 1992, 11.526, de 4 de outubro de 2007, 11.458, de 19 de março de 2007, e 12.350, de 20 de dezembro de 2010, e a Medida Provisória no 2.185-35, de 24 de agosto de 2001; e revoga dispositivos da Lei no 9.649 , de 27 de maio de 1998. Diário Oficial da União 2011; 10 ago.

9. Pessatti MP. Estratégias para a ambiência na humanização de Partos e Nascimentos. Brasília: MS; 2014. (Caderno Humanizasus)
10. Dias PF, Miranda TPS, Santos RP, Paula EM, Bem MMS, Mendes MA. Formação do conceito ambiência para trabalho de parto e parto normal institucionalizado. Rev. Bras. Enferm. 2019; 72(Supl. 3):348-359.

11. Agência Nacional de Vigilância Sanitária. Resolução RDC no 50, de 21 de fevereiro de 2002. Regulamento técnico para planejamento, programação, elaboração e avaliação de projetos físicos de estabelecimentos assistenciais de saúde. Diário Oficial da União 2002; 20 mar.

12. Brasil. Ministério da Saúde (MS). Portaria no 930, de 10 de maio de 2012. Define as diretrizes e objetivos para a organização da atenção integral e humanizada ao recém-nascido grave ou potencialmente grave e os critérios de classificação e habilitação de leitos de Unidade Neonatal no âmbito do Sistema Único de Saúde. Diário Oficial da União 2012; 11 maio.

13. Vilela MEA, Leal MC, Thomaz EBAF. Avaliação da atenção ao parto e nascimento nas maternidades da Rede Cegonha: os caminhos metodológicos. Cien Saude Colet 2021; 26(3):789-800.

14. Brasil. Portaria $\mathrm{n}^{\mathrm{o}} 1.020$, de 29 de maio 2013. Institui as diretrizes para a organização da Atenção à Saúde na Gestação de Alto Risco e define os critérios para a implantação e habilitação dos serviços de referência à Atenção à Saúde na Gestação de Alto Risco, incluída a Casa de Gestante, Bebê e Puérpera (CGBP), em conformidade com a Rede Cegonha a. Diário Oficial União 2013; 31 maio.

15. Costa JO, Xavier CC, Proietti FA, Delgado MS. Avaliação dos recursos hospitalares para assistência perinatal em Belo Horizonte, Minas Gerais. Rev Saude Publica 2004; 38(5):701-708.

16. Datasus. Sinasc - Sistema de Informações de Nascidos Vivos. Arquivos de declarações de nascido vivos. [acessado 2020 Jun 20]. Disponível em: http://www2.datasus.gov.br/DATASUS/index.php?area $=060702$

17. Leal MC, Bittencourt SA, Esteves-Pereira AP, Ayres BVS, Silva LBRAA, Thomaz EBAF, Lamy ZC, Nakamura-Pereira M, Torres JA, Gama SGN, Domingues RMSM, Vilela MAE. Avanços na assistência ao parto no Brasil: resultados preliminares de dois estudos avaliativos. Cad Saude Publica 2019; 35(7):e00223018.

18. Magluta C, Noronha MF, Gomes MAM, Aquino LA, Alves CA, Silva RS. Estrutura de maternidades do Sistema Único de Saúde do Rio de Janeiro: desafio à qualidade do cuidado à saúde. Rev. Bras. Saude Mater. Infant. 2009; 9(3):319-329.

19. Mendes A, Carnut L, Guerra LDS. Reflexões acerca do financiamento federal da Atenção Básica no Sistema Único de Saúde. Saúde debate 2018; 42(n. esp. 1):224243.

20. Brasil. Ministério da Saúde (MS). Sistema de Propostas do Fundo Nacional de Saúde (SISPROFNS), Sistema de Monitoramento de Obras (SISMOB), Gestão de Consultas e Normas (GESCON). Brasília: MS; 2019.

21. Silva CN. Ergonomia aplicada na qualificação da ambiência do espaço de nascer. Sustinere 2018; 6(1):150174. 
22. Rattner D. Humanização na atenção a nascimentos e partos: ponderações sobre políticas públicas. Interface (Botucatu) 2009; 13(Supl. 1):759-768.

23. Organização Pan-Americana de Saúde (OPAS). Recomendaciones de la OMS: cuidados durante el parto para una experiencia de parto positiva. Washington: OPAS; 2018.

24. Leal MC, Esteves-Pereira AP, Vilela MEA, Alves MTSSB, Neri MA, Queiroz R, Santos YRP, Silva AAM. Redução das iniquidades sociais no acesso às tecnologias apropriadas ao parto na Rede Cegonha. Cien Saude Colet 2021; 26(3):

25. Bittencourt SDA, Vilela MEA, Oliveira MC, Santos AM, Silva CKRT, Domingues R, Reis AC, Santos GL. Atenção ao Parto e Nascimento em Maternidades da Rede Cegonha: Avaliação do grau de implantação das ações. Cien Saude Colet 2021; 26(3):801-822.

26. Pasche DF, Righi LB. Apoio como estratégia de ativação do movimento constituinte do SUS: reflexões sobre a Política Nacional de Humanização (PNH). In: Campos GWS, Figueiredo MD, Oliveira MM. O apoio paideia e suas rodas: Reflexões sobre práticas em saúde. São Paulo: Hucitec; 2017. p. 141-166.

27. Vasconcelos MFF, Martins CP, Machado DO. Apoio institucional como fio condutor do Plano de Qualificação das Maternidades: oferta da Política Nacional de Humanização em defesa da vida de mulheres e crianças brasileiras. Interface (Botucatu) 2014; 18(Supl. 1):997-1011.

28. Brasil. Ministério da Saúde (MS). Humanização do parto e do nascimento. Brasília: MS; 2014.

29. Brasil. Ministério da Saúde (MS). Política Nacional de Humanização da Atenção e Gestão do SUS. A experiência da diretriz de Ambiência da Política Nacional de Humanização - PNH. Brasília: MS; 2017.
30. Paulon SM, Elahel ALS. A construção de um observatório de práticas da política nacional de humanização do Sistema Único de Saúde: a experiência do Rio Grande do Sul. In: Brasil. Ministério da Saúde (MS). Política Nacional de Humanização. Formação e intervenção. Brasília: MS; 2010. p. 141-156.

31. Campos GWS. Considerações sobre a arte e a ciência da mudança: revolução das coisas e reforma das pessoas. O caso da saúde. In: Cecílio LCO, organizador. Inventando a mudança na saúde. São Paulo: Hucitec; 1994. p. 29-86.

32. Pasche DF, Passos E, Hennington E. Cinco anos da política nacional de humanização: trajetória de uma política pública. Cien Saude Colet 2011; 16(11):45414548 .

33. Gomes MASM. Compromisso com a mudança. Cad Saude Publica 2014; 30(Supl. 1):S41-S42.

Artigo apresentado em 02/10/2020

Aprovado em 13/01/2021

Versão final apresentada em 15/01/2021

Editores-chefes: Romeu Gomes, Antônio Augusto Moura da Silva 